\title{
IITI Situación comunicativa y estructura genérica en la telenovela mexicana
}

RODNEY WILLIAMSON

UNIVERSIDAD DE OTTAWA

RESUMEN. A través de diferentes caracterizaciones teóricas del género discursivo se perfila la naturaleza compleja y dinámica de esta noción, tomándola como base de un modelo jerárquico de análisis del discurso de la celenovela mexicana. Se justifica la inclusion de las nociones de registro y de marco en el mismo modelo, ejemplificando el empleo de estos tres espacios semánticos para examinar la generación y la expresión de sentidos en una serie de niveles que van desde el macrotexto más amplio hasta los niveles microtextuales. Se comenta brevemente la articulación genérica y subgenérica de la telenovela, subrayando su inrerés para el escudio de los procesos de la semiórica social.

PALABRAS CLAVE: género discursivo, registro, marco, telenovela, semiótica social.

RESUMO. Através das diferentes caracterizaçôes teóricas do gênero discursivo, perfila-se a natureza complexa e dinâmica desta noção, quc se toma como base para um modelo gerárquico da análise do discurso da relenovela mexicana. Justifica-se a incluão das noçōes de registro e marco no mesmo modelo, exemplificando o emprego deste três espaços semânticos, con o fim de examinar a geração e a expressão de sentidos, numa série dc níveis que vão desde o macrotexto mais amplo até os níveis microtextuais. Comenta-se brevemente a articulação genérica e sub-gcnérica da telenovela, sublinhando seus interesses pelo estudo dos processos da semiótica social.

PALAVRAS CHAVE: gênero discursivo, registro, marco, telenovela, semiótica social. 
ABSTRACT. Different theoretical perspectives on the notion of discourse genre serve to underline the complex and dynamic nature of this concept. raken as the basis for a hierarchical model for analyzing the Mexican soap opera. We then justify the inclusion of the notions of register and frame in the same model through illustration of the use of all three semantic spaces to examine the generation and expression of meanings at a series of different levels, from the broadest mactotextual level right down to that of microcext. We comment briefly on the generic and subgeneric organization of the 'telenovela' or soap opera, emphasizing its significance for the srudy of social semioric processes.

KEY WORDS: discourse genre, register, frame, soap opera, social semiotics.

En este artículo nos hemos fijado un doble propósito: reflexionar sobre la noción de 'género discursivo' para luego aplicarla al análisis de unos resultados preliminares de un proyecto colaborativo entre El Colegio de México y la Universidad de Ottawa titulado "Discurso mediático y género: elementos visuales y verbales en la telenovela mexicana", en el que se enfoca el discurso relenovelesco. Este proyecto está asociado al megaproyecto dirigido por el Dr. Raúl Ávila en El Colegio de México sobre la "Difusión Internacional del Espańol en la Radio, la Televisión y la Prensa", en el que participan diecinueve países 1 .

La noción de 'género', ya liberado de su tradicional conceptualización formal y estática, con funciones meramente clasificatorias, ha conocido una interesante y rica evolución en los años 80 y 90 en el marco de los estudios mediáticos y como concepto clave en la semiórica social del lenguaje. En 1988 Hodge y Kress caracterizaron el género como "una categoría semiórica que codifica los efectos de los cambios sociales, de las luchas sociales" 2 , subrayando la naturaleza dinámica e intrinsecamente social del concepto, y su asociación imprescindible con un grupo social determinado. Afirmaron que "los géneros sólo existen en la medida en la que un grupo social afirme e imponga las reglas que los constituyen" 3 . Además, como entidades especificadas por sistemas logonómicos ${ }^{4}$, los géneros son importantes vehículos de ideología. En orras palabras, el 'género' constituye una práctica comunicativa y social en la que el acceso y los privilegios de emisores y receptores, y su entendimiento de los mensajes producidos, dependen de reglas y principios de autoridad y de procedimiento. Aunque Hodge y Kress no lo dicen explícicamente, la noción de género encierra una concepción de organización jerárquica interna.

Fairclough, por su parte, al comentar el uso del concepto en los estudios de los medios de comunicación y en contextos pedagógicos, traza su evolución como proceso de enriquecimiento progresivo desde 
una fase inicial de descripción "esquemática" hacia una perspectiva "heterogénea" y luego "polifónica" (Fairclough, 1995: 85-90). La fase "esquemática" se puede ilustrar, según él, con ejemplos de la lingüística sistémica o con el esquema formulado por William Labov (1972) para la descripción de las narraciones orales. Este género se articula en una serie de componentes ordenados y discretos: "abstract", orientación, hilo narrativo, desenlace, $y$ coda, por ejemplo, en el caso de las narraciones orales (aunque, como lo demuestra Labov, el componente evaluativo permea la narración entera). A los ejemplos mencionados por Fairclough, podríamos agregrar el concepto de género presentado por Hymes (1974) $y$ el interesante esquema de van Dijk para el análisis del reportaje periodistico que integra los diferentes componentes del reportaje en una jerarquía de cinco niveles (van Dijk, 1990: 86).

La perspectiva "heterogénea" es la que adopta van Leeuwen (1987) al enfocar las interesantes combinaciones de género que se dan en el discurso mediárico. Éstas sólo se pueden analizar partiendo de la red de estrategias comunicativas en juego, en vez de mirar sólo la organización textual. De esta manera se puede detectar cómo, en un artículo de la prensa, un periodista puede reportar opiniones y explicaciones expresadas por cierta autoridad sobre el tema, y a la vez exhortar al público lector a seguir ciertas recomendaciones o instrucciones. La tercera perspectiva, la "polifónica" emana de esce mismo carácter heterogéneo y múltiple que evidencian muchos discursos mediáticos, y a primera vista parece consistir simplemente en la constacación del conocido principio bajtiniano de la multiplicidad de voces en el discurso. Detectar polifonfa en un artículo periodístico o un programa televisivo en sí no significa más que consignar lo evidente, pero lo que aquí interesa es su ordenación jerárquica: en palabras de Fairclough, "the web of voices is often a subtle ordering and hierarchization of voices" (1995: 81). Para explicar el fenómeno en términos de Hodge y Kress $(1988,1993)$, el sistema logonómico hace que ciercas voces se legitimen $y$ orras no.

Por otra parte, en la concepción bajtiniana de la polifonía discursiva no sólo las voces entran en juego, sino también los estilos y los modos discursivos. Los estilos discursivos implican una relación específica entre emisor y receptor, los modos un canal o medio particular de comunicación (modos de transmisión oral o escrica, por ejemplo) (Fairclough. 1995: 71; Bakhtín, 1986). Una forma común de heterogeneidad de modos discursivos identificada por Fairclough es lo que llama la "conversacionalización": "the colonization of the discursive practices of the media by private domain practices, as a pervasive feature of the contemporary media" (1995: 89). Por ejemplo, el reportaje informativo o la entrevista con una figura pública o influyente, como prácticas discursivas pertenecientes al dominio público, se pueden entremezclar o modular 
con conversaciones personales. Salta a la vista que la polifonía y la "conversacionalización" son rasgos que caracterizan la telenovela, diferenciándola de muchos otros géneros narrativos.

Fairclough y otros señalan la heterogeneidad genérica como característica de los medios masivos de comunicación. Cabe preguntar también si la hibridación genérica sería típica de estos mismos medios. Es fácil buscar ejemplos de hibridación en toda una gama de programas desde los "talk shows" hasta los noticiarios televisivos, pero requerimos de muchos más estudios al respecto. En lo que concierne a la telenovela, la mezcla de formas narrativas y didácticas sería un punto interesante de análisis, sobre todo en países y contextos sociales en los que esta forma de entretenimiento se pone explícitamente al servicio de propósitos educativos o propagandísticos, por ejemplo. Hasta ahora, sin embargo, sólo hemos encontrado unos casos de hibridación tímida y limitada en nuestros materiales mexicanos.

La telenovela es una novela dialogada que conlleva una semiosis visual. Género que emana tanto de la tradición de la novela seriada como, por el lado dramático, del melodrama y del sainete, es diferente de ambos por ser un género consciente y voluntariamente moderno, apoyado en tecnologías modernas y un contexto comercial en el que se mira al televidente como consumidor. Las pausas entre escenas, y en muchos casos el ritmo mismo de la escenificación, se realizan en función de los anuncios comerciales que pueden incluso invadir el marco de la telenovela misma (los mismos personajes de la novela salen en anuncios, la música de la novela se anuncia para compra en $\mathrm{CD}$, etc.) Como narración seriada, la telenovela apunta hacia un final que con la proliferación de escenas, episodios y subtramas se va postergando, pero que llega inevitablemente. En este sentido su estructura relativamente cerrada contrasta con la de final abierto que identifica Robert Allen como típica del "soap opera" del mundo anglofono:

we need to distinguish between two fundamentally different, but frequently conflated, forms of relevision serial: what I call 'open' and 'closed' serials: US daytimc, British, and Australian serials are open narrative forms. That is to say they are the only forms of narrative (with the possible exception of comic strips) predicated upon the impossibility of ultimate closure. No one sits down to watch an episode of one of these programs with the expectation that this episode mighr be the one in which all individual and community problems will be solved and everyone will live happily ever after. (Allen, 1995:18).

Ahora bien, el final feliz, o por lo menos satisfactorio, es precisamence lo que espera el televidente latinoamericano:

Unlike the open serial, the Latin American telenovela and other forms of closed serial are designed to end and their narratives to close — although this closure might 
not be achieved until after several months or 200 episodes.

[...]

To Nick Vink, closure represents a key difference between Brazilian and North American serials. The teleological thrust of the telenovela privileges the final episodes institutionally, textually, and in terms of audience and satisfaction. The ending of a telenovela is heavily promored, and, in the case of particularly popular telenovelas, becomes the subject of anticipatory public and private discourse: "how will everything work our?" "Who will win and who will lose?" "Who will live and who will die?" (Allen, 1998: 23).

La estructura abierta de la "soap opera" le resta potencialidad como vehículo de mensajes ideológicos y juicios valorativos, convirtiéndola en cambio en foro de discusión de temas contenciosos o de actualidad:

The absence of a final moment of narrative closure also indefinitely postpones any moment of final ideological or moral closure in the open serial. This probably makes the open serial a poor vehicle for the inculcation of particular values, but it does mean that open serial writers and producers can raise any number of potentially controversial and contentious social issues without having to make any ideological commitment to them. (Allen, 1998: 21).

En cambio, el compromiso ideológico y el final moralizante son precisamente lo que ofrece la telenovela latinoamericana, a veces casi a la manera de la alegoría medieval. Comentando dos finales alternativos de una telenovela brasileña, una televidente escribió lo siguiente en una carta mandada al periódico: "Everyone knows," she wroce, "that in realicy the powerful never go to prison. Just for that reason at least a (cele) novela should offer this satisfaction." (Allen, 1995: 23). Como forma cerrada, la celenovela corresponde en el mundo anglófono, no a la "soap opera", sino más bien a las novelas policíacas estadounidenses, en las que, como compensación de la vida real, los policías se convierten en héroes y se hace la justicia.

De esta estructura cerrada de la telenovela, creemos que se desprende la posibilidad de analizarla aplicando un modelo jerárquico que relacione de manera sistemática y precisa su "micronivel" de escenas de intercambio conversacional entre personajes concretos con el "macronivel" de su articulación ideológica y su recepción y significación genéricas. Utilizamos como punto de partida el modelo provisional de género y registro que ofrecen McCarthy y Carter (1994: 36). Este modelo ofrece la ventaja de caracterizar el género a partir de sus funciones básicas y de incerrelacionar el mundo y los modos del discurso en roda su amplitud con los rasgos específicos de los registros linguísticos empleados a nivel microrextual. Parte del "mundo del discurso" que puede ser de naturaleza oral o escrita, dentro del cual se identifica una "función genérica básica" que motiva los diferentes "géneros" que una sociedad o comunidad determinada desarrolla para este propósito. Cada género puede realizarse en 
diferentes contextos reales y concretos en combinación con otros géneros, dando lugar a veces a hibridaciones o fusiones genéricas o, como en el caso nuestro de la telenovela, a combinaciones subgenéricas. Éstas a su vez se expresan en situaciones comunicativas específicas a través de "registros". McCarthy y Carter ilustran estos conceptos con el ejemplo de los géneros de reportaje (cuya función genérica básica es la de informar), cuyos casos concretos podrían ser, por ejemplo, el informe noticioso, un informe interino en algún proyecto, un boletín meteorológico, etc. En tales casos, el género de reportaje se combina con funciones de pronóstico, de evaluación, o de consejos o recomendaciones. La función del boletín meteorológico, por ejemplo, no es sólo informar sobre la situación actual y el pasado reciente sino también pronosticar el futuro. Esta combinación genérica se expresa a través de registros específicos referidos a determinados espacios geográficos, gamas de temperatura, etc. Y las modalidades precisas de su expresión variarán de acuerdo con su medio especifico de transmisión: televisión, radio, periódico, etc.

Adaptamos este modelo al caso de la telenovela en la forma que se muestra en la Figura 1. Para poder contemplar factores de generación de sentido a nivel individual (a nivel de parlamentos individuales de los personajes), incluimos el nivel de los "marcos", concepto que se explicará más adelante. En el caso de la telenovela, comenzamos con un mundo del discurso que parce de un instrumento escrito, el guión, pero que sólo se puede concretar y vehicular por medio del discurso oral de los personajes. No hay narrador como tal, o si lo hay, quiere ser un nasrador invisible e implícito. La función genérica básica es la narración, y siguiendo a Fairclough (1995) y Swales (1990) podemos definir la narración en sí como un "pre-género" o "protogénero" que define funcionalmente los diferentes géneros o variedades narrativas que se dan en contextos sociales reales.

El estudio de los 'registros' del lenguaje no está exento de problemas. Todavía no tenemos un perfil claro de su operación, y como señala de Beaugrande (1993) en una de las más perspicaces reseñas históricas del concepto, el 'registro' es un elemento perturbador en el marco de las abstractas reorias lingüisticas modernas. Su popularidad se debe esencialmente a Halliday 5 , quien lo define primero como un tipo de lenguaje seleccionado de acuerdo al tipo de situación (Halliday et al.,1964). Inicialmente se estudia de manera descriptiva, en términos exclusivamente léxico-gramaticales, pero a partir de los años 70 Halliday lo define como un concepto semántico. En 1978 nos ofrece la siguente definición:

[Register] is the set of meanings, the configuration of semanric patterns, that are typically drawn upon under the specified conditions, along with the words and structures that are used in the realization of these meanings (Halliday, 1978:23). 
FIGURA 1. MODELO JERARQUICO PARA EL ANALISIS DE LA TELENOVELA

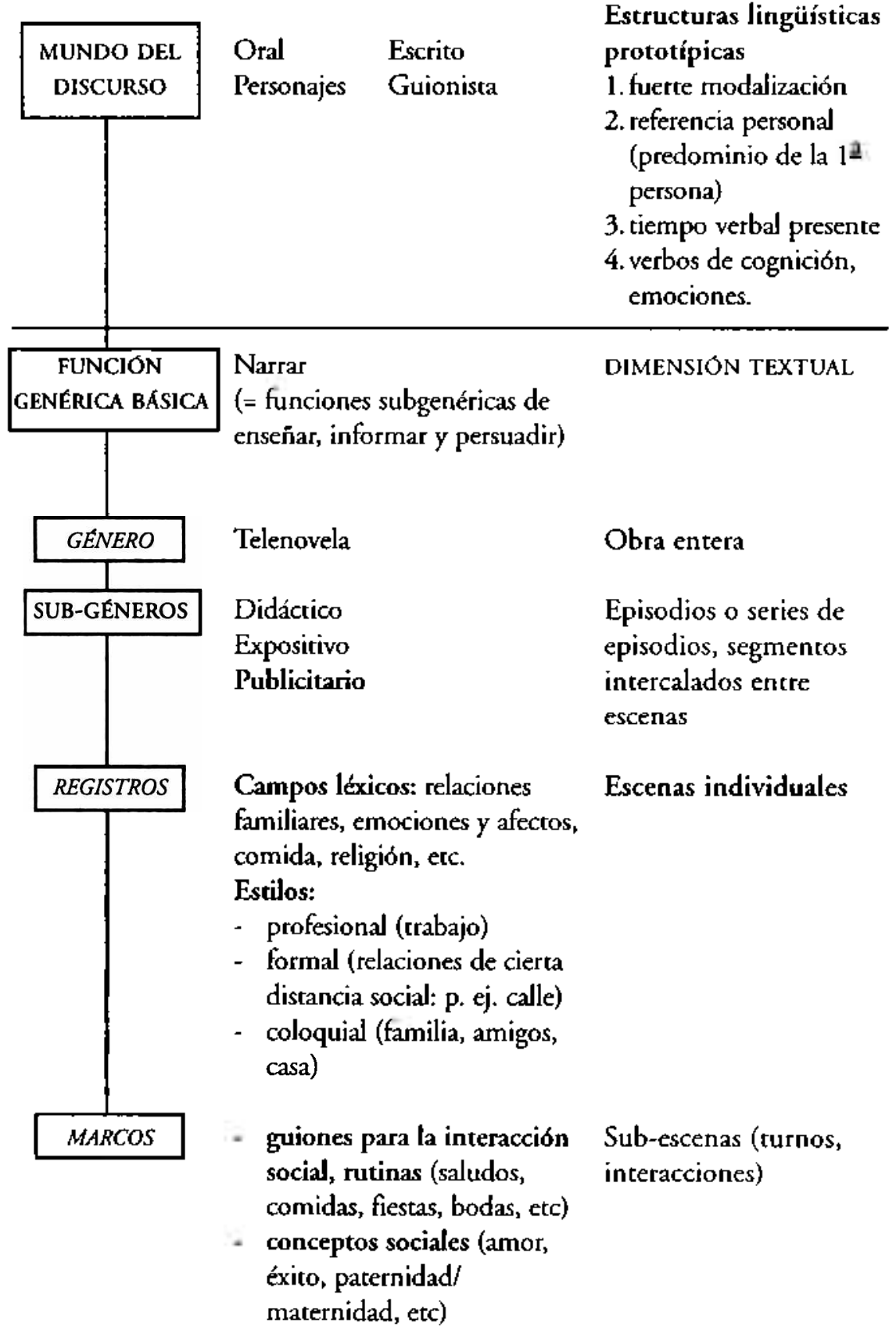


Y se fija no sólo en los resultados lingüísticos de este proceso de selección, sino cambién en el sujeto, el "miembro de una cultura", que lo realiza:

A register can be defined as the configuration of semantic resources that the member of a culrure rypically associates with a situation type. It is the meaning potential that is accessible in a given social context. (Halliday, 1978: 31).

Explorando el desarrollo posterior de éste y orras nociones afines, y destacando la importancia del concepto de "superestructura" de van Dijk, de Beaugrande llega a la conclusión de que el enfoque esencialmente sociológico desde el cual se ha estudiado el registro necesita complementarse con una perspectiva psicológica, ya que el registro tiene que ver con "a set of beliefs, attitudes or expectations about what is or is not likely to seem appropriate and be selected in certain kinds of contexts". (de Beaugrande, 1993:18).

Sin olvidar la importancia de los datos lingüísticos concretos y objetivos, hacemos eco de esta orientación hacia el sujeto ya que, en el caso de la telenovela, díficilmente se podría pasar por alto la parte que juegan los personajes, los locutores, en el texto que producen, y la interacción que se establece entre ellos. Hace falta enfocar la noción de "registro" en términos de procedimiento constructivo, y para eso nos parece útil echar mano de otra noción analítica: la de "frame" o "marco". El término "frame" también plantea problemas. Descartando la acepción banal de 'borde' o 'límite' de algo, se ha empleado con una gama de sentidos ligeramente divergentes, como si fuera una intuición común contemplada desde distintos ángulos o enfoques disciplinarios. Hoy día lo heredamos principalmente, primero, de Erving Goffman quien, como sociólogo, destaca su funcionalidad interactiva y lo aplica al análisis de la conversación en distintos contextos sociales; $y$ en segundo lugar, a Marvin Minsky y a otros especialistas de la inteligencia artificial, quienes lo conciben como un instrumento de la cognición y de las representaciones mentales. Minsky lo define como "una estructura de datos que sirve para representar una situación estereotípica" (1980:1), un esquema aprendido que el individuo aplica, apelando a su memoria, para simplificar las tareas de percepción y comprensión del mundo. Y si se aplica de esta manera a las tareas de recepción y de asimilación de datos y significaciones, se puede aplicar también a las tareas de producción: a la enunciación de mensajes lingüísticos en la interacción social. Esta doble funcionalidad cognitiva e interactiva es, en el fondo, algo que Goffman había entendido perfectamente. El marco es a la vez acción y comprensión: 
Organizational premises are involved, and these are something cognition somehow arrives at, not something cognition creates or generates. Given their understanding of what it is that is going on, individuals fit their actions to this understanding and ordinarily find that the ongoing world supports this fitring. These organizational premises asustained both in the mind and in activitya $l$ call the frame of the activity. (E. Goffman, Frame analysis: An essay on the organization of experience. Reproducido en Lemert y Branaman, 1997: 158).

Si bien en esta cita parece descartarse el papel generador de la mente para transformar la experiencia, hacia el final del mismo ensayo Goffman apunta que: "in countless ways and ceaselessly, social life takes up and freezes into itself the understandings we have of it" (en Lemert $y$ Branaman, 1997: 163).

Entre los estudios que aplican el concepto de "frame" al análisis lingüístico se destacan los de van Dijk (1977) y Deborah Tannen y sus colaboradoras (Tannen 1984, 1993) ${ }^{7}$. Estas últimas parecen dar más peso a su lado interactivo, como indicador de diferencias culturales. A decir verdad, el "marco" es indicador de semejanzas también: como instrumento cognitivo, nos simplifica el mundo, nos revela lo que es "lo mismo" o idéntico entre nuestras experiencias y en las de otras personas. $\mathrm{Y}$ como instrumento de interacción, nos remite a las inevitables diferencias entre los seres humanos, a la infinita variedad del mundo. Aquí faltaría espacio para entrar en todos los pormenores de nuestra formulación de los marcos. Digamos sencillamente que nos parece importante asimilar lo mejor de las diferentes fuentes citadas: Minsky (1980) nos hace apreciar las ventajas de una rigurosa descripción de la estructura interna del marco; Goffman (1974) nos sensibiliza ante la preeminencia de los marcos interactivos y de los que hacen avanzar una acción; Tannen (1993) nos sefiala cómo los marcos se aplican a la tealización de tareas comunicativas y a la interpretación de estructuras linguísticas. Por otra parte, debemos tener presente que los marcos presentan un doble peligro en la investigación empirica: en primer lugar, es posible considerar el marco como principio constructivo de casi cualquier conceptualización y de ahí, como investigadores, "inventar" los marcos necesarios para explicar nuestros datos; en segundo lugar, en la medida que los marcos incluyen contenidos individuales, es posible imponer nuestras interpretaciones personales desvirtuando así las conceptualizaciones de otros.

Con afán de evitar estos peligros, presentaremos algunos casos de los marcos más evidentes en nuestros materiales, que hasta la fecha provienen de dos telenovelas grabadas en 1997: "Mirada de mujer" de TV Azteca, y "Desencuentro" de Televisa. Reviste especial interés la primera por querer romper con algunos de los estereotipos del género, al presentar una perspectiva femenina sobre temas de actualidad de especial 
relevancia para el medio de clase media urbana que representa. Algunos de éstos muestran hibridación de discursos: al tratarse el tema del acoso sexual, por ejemplo, se entremezcla un discurso público, incluso con estadisticas, en las discusiones personales de los personajes. Incluso hay inversión de roles, como cuando el personaje masculino principal se ve obligado a contestar las preguntas de un cuestionario sobre el amor que aparece en una revista de mujeres que está leyendo su secretaria. Y el personaje central es una mujer que, ante la infidelidad de su marido, se atreve a vivir una relación con un hombre diecisiete años menor que ella.

En "Mirada de mujer" es notable que, por una parte, muchos marcos se establecen interactivamente $y$, por otra, que sirven para caracterizar conflictos sociales que se realizan principalmente sobre dos ejes: el que opone el hombre a la mujer, y el que establece diferencias y contiendas entre jóvenes y mayores. En el ejemplo 1, los conocimientos "ilustrados" y cosmopolitas de Alejandro sobre la comida entran en conflicto con el esquema simple del "fast food" y películas rentadas de su joven hijo Alex:

Ejemplo 1

$<$ AlexM> ¿Qué es esto, papá?

<Alejandro> Es comida japonesa. ¿Qué? No, no hagas gestos. Primero pruébala y después haces gestos.

<Alex> Preferirfa una pizza o una hamburguesa.

<Alejandro> Sí, tú preferirías pizza o hamburguesa, pero antes de que venga tu mamá o una trabajadora del DIF a llevarte a un albergue porque tú papá no te da comida sana prueba las cosas y luego te quejas.

$<$ Alex> Y esto ¿qué es?

<Alejandro> Es sushi, se llama sushi.

$<$ Alex> ¿Qué tiene?

<Alejandro> Mira, mira, tiene pescado crudo, alga, queso, ajonjolí y esto es como jengibre, creo que se llama. Sabe como a perfume y esto es una raíz fértil que es como el chile, pero no sabe como el chile, pero es muy fuerte.

$<$ Alex> iAh! de verdad.

$<$ Alejandros Suena mal, pero sabe muy bien.

$<$ Alex> Mira, yo tengo un amigo que es de Japón y alli comen perro y gato y esas cochinadas como aqul comemos nosotros vaca y cerdo.

(Dos escenas más adelante)

Alejandro $>$ Hubiera comprado una pizza grande y rentado una película. <Alex> ¡Ajá! y ¿por qué no me hiciste caso? te llevo diciendo eso hace media hora.

<Alejandro> Ya, ya cállate, deja de acariciar la comida y vece a jugar nintendo, ándale. 
Es interesante notar aqui la reclamación de Alex al final: "te llevo diciendo eso hace media hora". La pizza no la ha mencionado sino una sola vez, y la película ninguna. Lo que si ha estado diciendo es que los japoneses comen cosas diferentes que "nosotros", que por lo tanto son "cochinadas". Como es típico de los marcos, sólo aparecen parcialmente en la enunciación, pero ni Alejandro ni nosotros los espectadores tenemos la más mínima dificultad en comprender el marco que en este caso está manejando Alex hijo. La comida en ambas telenovelas es uno de los símbolos más evidentes que diferencian individuos y clases sociales, $y$ en el caso citado los marcos se reflejan lingüísticamente sobre todo en sustantivos y estructuras atributivas (parte de las relacionales, según Hodge y Kress (1993). Éste es el contenido típico de los marcos que ponen en juego juicios sobre cosas.

Muy diferente contenido lingüístico arrojan los marcos telativos a! tema del matrimonio. En "Mirada de mujer", Andrés, el hijo de la protagonista María Inés, se casa con su novia negra, Jvana, sin que ellos digan nada a sus respectivos padres. Veamos primero la reacción de los padres de Ivana:

Ejemplo $2 A$

$<$ Padre> ¿Que hiciste que?

<Ivana> Me casé//No te vayas, mamá. (la agarra para que no se vaya de escena)

<Madre> Necesito pensar para no lamentarlo./Te dimos toda nuestra confianza, ¡Ivana! Te apoyamos. Jamás te negamos nada. ¿No crees que debiste.. consultarnos, o, al menos, avisarnos?

$<$ Padre> ¡Irresponsables! ¡Son un par de irresponsables!

$<$ Madre> Marcel, ipor favor!

$<$ Ivana> Por eso. Sabía que no lo entenderlan.

<Padres Claro que no. No era el momento ni la persona indicada.

$<$ Ivana> Amo a Andrés. Ninguno es tan maravilloso como él.

$<$ Padre> Sí, un músico. Que no podrá ganarse más del salario minimo.

Un joven que tiene que terminar sus estudios para responsabilizarse de una familia./Que lo amas. Eso no es amor, eso es un capricho de tu parte.

$<$ Madre> Apenas lo conoces.

$<$ Padre> Lo conoce muy bien, porque pracricamente ha estado viviendo

en su departamento./Me defraudaste, Ivana.

$<$ lvana $>$ Eso no es cierco. Debí decirte, pero...

$<$ Padre> Pero no lo hiciste.

$<$ Ivana> Mamá...

$<$ Madre> ¿Estás embarazada?

$<$ Ivana> ¡No! No me acosté con Andrés antes de casarme. Era virgen. 
Es evidente en este caso que el marco que maneja el padre de Ivana se ha violado, y que ella no ha respondido a sus expectativas. En términos lingüísticos se destaca en este intercambio la presencia de los verbos, que son quizá incluso más importantes que los sustantivos en el funcionamiento del marco. Esto se debe a que este tipo de marco, a diferencia del anterior, implica expectativas sobre una serie de acciones típicas o "normales". Es el tipo de marco que Schank y Abelson (1977) denominaron "guión ("script"), ilustrado en los ejemplos de análisis textual de van Dijk (1977). Lo que los mayores esperan de los hijos que contemplan casarse es más o menos lo siguiente:

1. Terminar los estudios

2. Establecerse económicamente

3. Tener planes para el futuro, incluyendo la posibilidad de tener familia

4. Comunicar sus intenciones a los padres

Y, en el caso de la novia:

5. Abstenerse de relaciones sexuales antes del matrimonio

6. Casarse de blanco y que el padre la "entregue" frente al altar.

Los pasos 1, 2 y 3 significan ser "maduro", "responsable" y demostrar "amor", y el no cumplirlos es ser "joven" e "irresponsable" y librarse a un "capricho". Faltar al número 4 es atentar contra la "confianza" y el "apoyo" de los padres, y la única explicación razonable de ello serla el no cumplimiento de 5, con el resultado de un embarazo no previsto. El marco de los jóvenes es, de nuevo, bastante más sencillo:

\section{Nos amamos}

2. Por ende nos podemos casar.

Nórese que para los mayores el 'amor' es un sustancivo, en tanto que los jóvenes emplean el verbo 'amar'.

Como en el ejemplo 1, encran en conflicto marcos de jovenes y mayores, pero ahora no sólo en los intercambios conversacionales de dos individuos sino a nivel de familias enteras. En los ejemplos 2B, C, D y E vemos hasta qué grado hay coincidencia entre los mayores en la enunciación del marco. 2B y 2D nos dan el intercambio entre Andrés y su madre, y $2 \mathrm{E}$ con su padre, en tanto que $2 \mathrm{C}$ retoma la conversación entre Ivana y su padre. Tanto el padre de Ivana como la madre de Andrés hablan del futuro, y mientras ésta habla de "planes" y "oportunidades", Andrés y su padre hablan de "proyectos". El padre de Ivana le reprocha por "defraudarlo", el de Andrés por "engañarlo". El padre de Ivana se 
refiere a Andrés como "un joven" y "ese muchacho"; el de Andrés se refiere a Ivana como "esa jovencita". Ambos coinciden en hablar de la madurez. Y los padres de Andrés, a pesar de todas las diferencias de carácter que tienen, se sienten ambos "decepcionados". En este caso, vemos cómo los marcos contribuyen a la vez a las conflictivas interacciones a nivel local, y al macronivel de articulaciones ideológicas: no sólo están en juego normas de comportamiento, sino principios de autoridad. Y un solo marco puede dirigir el progreso de la acción no sólo de una escena a otra, sino durante episodios enteros.

Ejemplo $2 B$

<María Inés> Pero, pero ¿por qué lo hiciste, Andrés?, ¿por qué?

$<$ Andrés> Porque la amo...

$<$ María Inés> ¿Y el amor iba a desaparecer si se esperaban?/iTenían la VIDA por delante!

[...]

$<$ María Inés> ... Ahora yo te pregunto: ¿ustedes dos lo pensaron, o fue una decisión que tomaron así, nada más, inconscientemente?

$<$ Andrés> No, no, lo pensé, lo lo pensamos.

<María Inés> Ah, ¿lo pensaron?

<Andrés> Sí, sí, lo pensamos. Pero no nos importa. Ni en lo más mínimo nos importa.

Ejemplo $2 C$

<Ivana> Lo importante es que Andrés y yo nos amamos.

$<$ Padre $>$ Ese muchacho es un "bueno para nada".

$<$ Ivanas Andrés, se llama Andrés y es mi marido.

$<$ Padre> Andrés. Tú crees que es lo suficientemente maduro como para enfrentar lo que vendrá de ahora en adelante?

<Ivana> Sí, sí lo es.

<Padre> Bueno, y ¿por qué no lo hizo cuando tenía que hacerlo?. ¿Tú crees, hija, que va a procurarte el nivel de vida al que estás acostumbrada?

\section{Ejemplo $2 D$}

<María Inés> ¡Ay, Andres!/Hijo ... tú tenías, tú tenías tantas oportunidades. Pero, en fin, ¿cuáles son rus planes ahora?

$<$ Andrés> Tengo muchos proyectos, pero luego te cuento.

$<$ María Inés>Es que.. Bueno, ya, cotal, ya to interesa.

$<$ Andrés> ¿Estás molesta conmigo?

<María Inés> Sí. No, no estoy molesta. Bueno, sí. No, no, no sé. No sé.

Me siento... es que estoy... Hijo... Tan decepcionada, Andrés. Tú te das cuenta que teníamos una relación maravillosa, una comunicación 
muy abierta, tú y yo. Si tenías intenciones de tomar decisiones tan importantes en tu vida, ¿por qué no te tomaste la molestia de, de avisarme, de comentarlo conmigo, de que lo pudiéramos discutir?

Ejemplo $2 E$

$<$ Padre de Andrés> Hasta el apoyo que te di para que vivieras en tu propio departamento y te concentraras en tu música fue lo último que hice por ti. Me engañaste, Andrés. Me hiciste creet que efectivamente habías comenzado a madurar, pero ¡qué esperanzas! Tu rebeldía no tiene límites, pero yo sí. Andrés, tu matrimonio con esa jovencita es algo que no puedo aceptar, aunque a decir verdad no me preocupa demasiado. En seis meses o un ańo la dejarás como has abandonado todo en tu vida. Has demostrado una absoluta incapacidad para llevar a cabo cualquier proyecto importante y yo no puedo hacer más de lo que ya he hecho por ti. Me tienes cansado, Andrés, muy cansado. Estoy muy decepcionado de ti.

Los ejemplos que hemos examinado hasta ahora muestran cómo los marcos contribuyen a la continuidad de la interacción, pero hay otros que provocan rupturas. Éstos casi siempre tienen una función ideológica especial, ya que la interrupción del flujo comunicativo llama la atención del espectador sobre ellos. En el ejemplo 3 vemos cómo uno de los marcos principales de "Mirada de mujer", la concepción femenina del hombre, es empleado como estrategia de ruptura por Paulina, la mejor amiga de María Inés. Después de las vociferaciones de Paulina contra el infantilismo de los hombres, Alejandro la interrumpe con sus "diez mandamientos" (que en realidad son muchos deseos y un par de responsabilidades). Paulina luego retoma la palabra remedando el típico discurso masculino, creando desconcierto y descontrol en Alejandro:

Ejemplo 3

$<$ Paulina> "¿Será qué escoy haciendo lo correcto? ¿será que estoy educando bien al niño? María Inés, ¿qué haremos con nuestro hijo?".I Hombre, tenías que ser hombre!! Necesitas una mamá, hasta para ser papá!!

<Alejandro> Se acabó, se acabó, se acabó! Sí, que se acabó! Estoy hasta la (censurado) ...

Mira, uno: amo a María.

dos: la amo por encima del bien, del mal, y de mi futura suegra.

tres: no me importa si es flaca, gorda, alta, bajica, anciana o adolescente.

cuatro: quiero tenerla las 24 horas del día, y no el tiempo que le deje libre su familia. 
ci-cinco: estoy dispuesto a aceptar a su familia incluyendo a la adorable Mónica.

cinco a.: Ojalá Mónica se case muy pronto para que tenga que soportarla el marido, no yo.

seis: quiero que María sea mi esposa.

siete: quiero ser el esposo de María.

ocho: No voy a estar tranquilo hasta que Alejandro Salas sea el marido de María Inés de Salas

nueve: quiero que seas mi testigo de bodas.

diez: tengo un hijo!!

Esos son mis diez mandamientos, son diez, no los puedo dejar en nueve.

$<$ Paulina> "De"

$<$ Alejandro> "De, de de...". ¿De que, "de"?

$<$ Paulina> Tenías que ser hombre, Alejandro. "De". "El carro nuevo de Alejandro". "La hermosa casa del señor Salas". "María Inés de Salas"... $\mathrm{El}$ que inventó el idioma español también era hombre. Mira, Alejandro: si pierdes a María Inés te voy a mandar a comer raíces de lirio y a empujar margaritas. ¿Está claro?

Terminamos con un ejemplo de la otra telenovela, "Desencuentro", para mostrar cómo un marco, en este caso el del enamoramiento tal y como se plantea en el discurso masculino, de forma jocosa, puede motivar un registro coloquial y altamente metafórico, no sólo en el léxico, sino también en la selección de estructuras sintácticas:

\section{Ejemplo 4}

<Luis> Anoche Victoria le llevó arroz con leche a la Maru y esraba delicioso.

$<$ Tony> Uy si... (cancando) arroz con leche me quiero casar...

$<$ Luis $>$ ¡Ya payaso!

$<$ Tony> El que se está viendo payaso eres tú, mano. No se te cae de la boca su nombre jVictoooria!

$<$ Luis> Pues ni que fuera taco al pastor para caerse de la boca, la mera verdad me encanta me fascina y que...

$<$ Tony> ¿Qué bueno mano! Desde que enviudaste nunca te había movido el tapete ninguna chava.

<Luis> Si, pero ya ves compadre, mi simoncita (refiriéndose al carro de bomberos), en quién me fui a fijar de bruto.

$<$ Tony> En una muchacha muy guapa.

$<$ Luis $>$ S1.

$<$ Tony> Y por lo que dices con todas las cualidades.

$<$ Luis> Sí muy guapa y con cualidades y ahora es tan pobre como yo pero antes no, era de otra clase. 
<Tony> (canta) Mi sangre aunque plebeya también se tiñe de rojo... $<$ Luis> Tony eres muy desafinado compadre, chíflale mejor. Suena horrible hombre, además que se supone que eres mi amigo, ve nada más puras burlas.

<Tony> Te equivocas, yo te apoyo. No sé cómo pero te apoyo. Soy bombero y tengo que apagar ese fuego de tu corazón.

En conclusión, esperamos haber demostrado con estos pocos ejemplos que los marcos no son meras proyecciones personales sino importantes elementos de interacción que crean vínculos entre personajes, escenas, hasta episodios enteros. Como vehículos de ideología contribuyen, en última instancia, a la articulación y el propósito de la obra entera. Sólo un modelo dinámico y jerárquico del género puede dar cuenta de este proceso constructivo y de sus repercusiones en distintos niveles.

Terminaremos con una advertencia. Los marcos altamente estereotipados, y los intercambios conversacionales mismos de las telenovelas, no se deben tomar por ejemplos verídicos de habla vernácula. Pero a pesar de su absoluta falta de autenticidad, la telenovela logra curiosamente una verosimilitud que no se pierde ni en los casos de mayor exageración. John Fiske lo explica como el "willing suspension of disbelief" del televidente, que se debe a su propia parricipación en el proceso de producción y representación, a una noción de cextualidad televisada fundamentada en una delegación de privilegios autoriales al espectador, en fin, su "democracia semiótica":

There is a pleasure in playing with relevision's textuality, in exploiting one's abilicy to submit oneself to, and to discance oneself from, irs illusion of reality that is finally a democratic one, for it allows the viewer both concrol of reading relations and access to the process of representation. (Fiske, 1989: 71).

La telenovela como modelo evidente y manejable nos ofrece un instrumento útil y cómodo para la exploración de los complejos procesos de la semiótica social. Por su extraordinario éxito en Latinoamérica y en el mundo entero es un fenómeno sociocultural en sí, cuyas características lingüísticas y discursivas merecen escudiarse.

\section{NOTAS}

I. Agradecemos al Dr. Avila su generosa ayuda y colaboración, y el acceso que nos ha dado a los materiales de su proyecto, encre los cuales se cuentan los de "Mirada de mujer" y "Desencuencro" que analizamos en este artículo.

2. R. Hodge \& G. Kress, Social semiotics (Cambridge: Polity Press, 1988: 7) "Genre therefore represents one semiotic category that codes the cffecrs of social change, of social struggle." La traducción al español es mía.

3. Ibid. La traducción es mía.

4. Op. cit., p.2ff. 
5. Aunque la noción hue utilizada por primera vez en 1956 por Thomas Reid, y desarrollada posteriormente por Jean Ure. La conceptualización de Halliday también debe algo a los trabajos de Basil Bernstein, como señala de Beaugrande (1993:9).

6. Goffman, a su vez, toma la noción de Bateson (cf. Bateson 1972), con quien reconoce tener bastances afinidades.

7. Para una reseña más completa y satisfaccoria de la cuestión que la que podemos dar aquí, véase el primer capitulo de Tannen (1993).

\section{NEFERENCIAS BIBLIOGRAFICAS}

ALLEN, R. C. (Ed.). (1995). To be continued... Soap operas around the world. Londres: Routledge.

BAKHTIN, M. (1986). Speech genres and other late essays. C.Emerson y M. Holquisr, Trad. Austin: University of Texas Press.

BATESON, G. (1972). A theory of play and phantasy. Artículo de 1955 reproducido en Steps to an ecology of mind (pp. 177-193). New York: Ballannine Books.

de Beaugrande, R. (1993). Register in discourse studies. En Mohsen Ghadessy (Ed.), Register analysis. theory and practice (pp. 7-25). Londres: Pinter Publishers.

FarRClOUGH, N. (1995). Media discourse. Londres: Edward Arnold.

FISKE, J. (1989) Moments of television: neither the text nor the audience. En Ellen Seiter et al. (Eds.), Remote control. television, audiences and cultural power ( $\mathrm{pp}$. 56-78). Londres: Roucledge.

GOFFMAN, E. (1974). Frame analysis: An essay on the organization of experience. New York: Harper \& Row.

Halliday, M. A.K.; Mclntosh, A. y Strevens, P. (1964). The linguistic sciences and language teaching. Londres: Longman.

Halliday, M. A.K. (1978). Language as social semiotic. Londres: Edward Arnold.

Hodge, R. y Kress, G. (1988). Social semiotics. Cambridge: Polity Press.

HODGE, R. y Kress G. (1993). Language as ideology. 2a. edición. Londres: Routledge. HYMEs, D. (1974). Foundations in sociolinguistics: An ethnographic approach. Philadelphia: University of Pennsylvania Press.

LABOV, W. (1972). Language in the Inner City: studies in the black English vernacular. Philadelphia: University of Pennsylvania Press.

Lemert, Ch. y Branaman, A. (Eds.). (1997). The Goffman Reader. Oxford: Blackweli.

MCCARTHY, M. y CARTER, R. (1994). Language as discourse: Perspectives for language teaching. Londres: Longman.

MinskY, M. (1980). A framework for representing knowledge. En Dierer Merzing (Ed.), Frame conceptions and text understanding (pp.1-25). Berlin: Walcer De Gruycer.

REID, T. B. (1956). Linguistics, structuralism, philology. Archivum Linguisticum 8.

SCHANK, R. C. y ABELSON, R. P. (1977). Scripts, plans, goals and undentanding: An inquiry into buman knowledge seructures. Hillsdale, NJ: Erlbaum.

SWaLES, J. (1990). Gente andysis: English in academic and research settings. Cambridge: Cambridge University Press.

TANNEN, D. (Ed.). (1984). Coherence in spoken and written discourse. Advances in discourse processes, Vol. XII. Norwood, NJ: Ablex.

TANNEN, D. (Ed.). (1993). Framing in discourse. Oxford: Oxford University Press. 
UrE, J. (1971). Lexical density and register discourse formation. En G.E. Perren y J.L.M. Trim (Eds.). Applications of linguistics. Cambridge: Cambridge University Press.

VAN DIJK, T. A. (1977). Text and consext. Londres: Longman.

VAN DıjK, T. A. (1990). La noticia como discurso. (Traducción de News as discourse, 1980) Barcelona: Paidós.

VAN Leeuwen, T. (1987). Generic strategies in press joutnalism. Australian Review of Applied Linguistics 10 (2), 199-220.

RODNEY WILLIAMSON es profesor de lingüistica hispánica y traducción de la Universidad de Ottawa. Ha publicado mayormente en las áreas de análisis discursivo y textual de textos hispánicos, y en la enseñanza del español. Actualmente trabaja en proyectos de colaboración internacional sobre la telenovela mexicana y la prensa mexicana y canadiense. Los temas por los que se interesa especificamente son la evolución de los géneros discursivos, la expresión de la ideología en el discurso, y la multimodalidad.

Correo electrónico: fonte@xanum.uam.mx 